\title{
El álgebra temprana en los libros de texto de Educación Primaria: implicaciones para la formación docente
}

\author{
Early algebra in primary education textbooks: implications for teacher \\ education
}

\author{
Nataly Pincheira* \\ ORCID iD 0000-0002-5051-964X \\ Ángel Alsina** \\ ORCID iD 0000-0001-8506-1838
}

\begin{abstract}
Resumen
En este estudio se analizan las tareas de álgebra temprana en una colección de ocho libros de texto de Educación Primaria chilenos, de amplia difusión. Para la investigación se ha seguido una metodología cualitativa, de carácter descriptivo, a partir de la técnica de análisis de contenido. Los resultados obtenidos muestran una variedad de tareas matemáticas con una distribución no homogénea, predominando las tareas de relaciones basadas en reglas, más específicamente, tareas que involucran la comprensión de patrones, seguidas de tareas de relaciones conocidas-desconocidas y una presencia menor de tareas de relaciones aritméticas situadas. Se concluye que es necesario brindar experiencias de formación al profesorado que les permita, por una parte, profundizar en el análisis de tareas matemáticas que promuevan el desarrollo del pensamiento algebraico temprano para alcanzar una adecuada selección e implementación de dichas tareas y, por otra parte, indagar en la actividad matemática que deben desarrollar los estudiantes para su resolución.
\end{abstract}

Palabras clave: Álgebra temprana. Enseñanza. Tareas matemáticas. Libros de texto. Educación Primaria.

\begin{abstract}
This study analyses the early algebra tasks present in a set of eight Primary Education Chilean textbooks of wide dissemination. The methodology used involves a qualitative approach of descriptive character, by means of the content analysis technique. The results obtained show a variety of mathematical tasks with a non-homogeneous distribution, with a predominance of rule-based relations tasks, more specifically, tasks involving the understanding of patterns, followed by known-unknown relations tasks and a minor presence of arithmeticallysituated relations tasks. It concludes that it is necessary to provide training experiences to teachers that allow them to deepen in the analysis of mathematical tasks that promote early algebraic thinking to achieve an adequate selection and implementation of such tasks on the one hand, and on the other, to investigate the mathematical activity that students must develop for its resolution.
\end{abstract}

Keywords: Early algebra. Teaching. Mathematical tasks. Textbooks. Primary Education.

\footnotetext{
* Doctoranda en Ciencias de la Educación por la Universidad de Girona (UdG), Girona, España. E-mail: nataly.pincheira@udg.edu.

** Doctor en Psicología por la Universidad Autónoma de Barcelona (UAB). Catedrático de Didáctica de las Matemáticas en la Universidad de Girona (UdG), Girona, España. E-mail: angel.alsina @udg.edu.
} 


\section{Introducción}

La introducción de conocimientos de naturaleza algebraica, desde los primeros niveles escolares, se ha impulsado durante las últimas dos décadas y, progresivamente, se ha ido asentado en los currículos de matemáticas de Educación Primaria de diversos países (e.g. ACARA, 2015; MINEDUC, 2012; MINISTRY OF EDUCATION, REPUBLIC OF SINGAPORE, 2012; NCTM, 2000).

Esta nueva alternativa de cambio e integración curricular se alberga bajo las directrices del enfoque Early-Algebra, a partir de ahora álgebra temprana, cuyo propósito es promover el desarrollo del pensamiento algebraico desde los primeros niveles de escolaridad y facilitar una mejor comprensión de las matemáticas (BASTABLE; SCHIFTER, 2007; CAI; KNUTH, 2011; CARRAHER; SCHLIEMANN, 2007; KAPUT, 2000). Más específicamente, los objetivos que persigue el álgebra temprana consideran el desarrollo de modos de pensamiento que permitan alcanzar una comprensión más profunda y compleja de la matemática escolar (BLANTON; KAPUT, 2005), dado que el estudio del álgebra se encuentra implícito, de manera transversal, en el currículo de matemáticas desde los primeros niveles (CARRAHER; SCHLIEMANN, 2007).

La incorporación del álgebra temprana en los currículos contemporáneos de matemáticas de Educación Primaria interpela directamente al profesorado, desafiándolos a desarrollar prácticas de enseñanza que consideren la selección pertinente e implementación de tareas matemáticas que promuevan, de manera paulatina y sistemática, la enseñanza de estos contenidos. En este contexto, el profesorado sustenta y guía sus prácticas de enseñanza apoyándose en diversos recursos, siendo el libro de texto una de las herramientas más recurrentes (SHIELD; DOLE, 2013).

Los libros de texto constituyen una fuente de información para el alumnado y el profesorado, con tareas de clase y preguntas de evaluación (CAMPANARIO, 2001), convirtiéndose en uno de los elementos más emblemáticos del proceso de enseñanzaaprendizaje (RAMÍREZ, 2003). Diversos estudios (e.g. PORTER, 2002; TARR et al., 2006) han informado que, muchas de las decisiones que toma el profesorado acerca de los conocimientos matemáticos que deben enseñar, y cómo enseñarlos, están influenciadas por los libros de texto que utilizan y, en consecuencia, la instrucción en el aula depende en gran medida de este recurso. Esto implica que las oportunidades de aprendizaje de los estudiantes estén estrechamente vinculadas con los conocimientos que promueven los libros de texto de matemáticas (STYLIANIDES, 2009). 
Adicionalmente, el uso del libro de texto por parte del profesorado supone replantearse o cambiar aspectos de la propia práctica docente (REMILLARD, 2000), puesto que ofrece oportunidades para utilizar, adaptar y comprender el trabajo de los estudiantes en las tareas matemáticas que sugieren. Desde este punto de vista, el libro de texto puede influir en la formación docente, es decir, en el conocimiento del profesorado para enseñar matemáticas y, en consecuencia, se debería prestar especial atención a las tareas matemáticas propuestas en este recurso para promover una enseñanza efectiva del álgebra temprana.

Considerando, por un lado, el impacto del libro de texto como herramienta para suscitar la enseñanza de las matemáticas y, por otro, que algunos estudios han evidenciado que las tareas de los libros de texto no están intencionalmente dirigidas al desarrollo del pensamiento algebraico temprano (AKÉ; GODINO, 2018), nuestro propósito es seguir investigando en esta dirección, para aportar nuevos datos que permitan una mayor comprensión tanto de las características que presentan las tareas relacionadas con el álgebra temprana en los libros de texto de Educación Primaria como de los conocimientos matemáticos que promueven dichas tareas para conducir la enseñanza de este bloque de contenido.

Para llevar a cabo este análisis, se integra el marco analítico de Demosthenous y Stylianides (2014) para determinar las tareas sobre álgebra temprana en los libros de texto de Educación Primaria, con la caracterización de los conocimientos matemáticos involucrados en la resolución de las tareas que promueven el desarrollo del pensamiento algebraico temprano de Pincheira y Alsina (2021). Con base en este marco, el objetivo de nuestro estudio es analizar las tareas sobre álgebra temprana presentes en una serie de ocho libros de texto escolares chilenos de Educación Primaria (6-12 años).

\section{La enseñanza del álgebra temprana en Educación Primaria}

La enseñanza del álgebra temprana en Educación Primaria se ha ido consolidando durante los últimos años, pues existe un consenso cada vez mayor de los investigadores por brindar experiencias de aprendizaje que fomenten el desarrollo del pensamiento algebraico desde los niveles iniciales (BLANTON; KAPUT, 2005; CARPENTER; FRANKE; LEVI, 2003; MOLINA, 2009; NCTM, 2000).

Incorporar el álgebra temprana en Educación Primaria requiere atender a una amplia concepción del álgebra que comprende diversos elementos, como la generalización de la aritmética, el estudio de las relaciones numéricas y la generalización de patrones, el estudio de 
las relaciones funcionales, el estudio de las estructuras matemáticas comunes abstraídas de cálculos y relaciones, junto con el desarrollo de la modelización y la resolución de problemas (KAPUT, 2000; MOLINA, 2011; MOLINA; CAÑADAS, 2018; SCHLIEMANN et al., 2003).

Diversas investigaciones informan que los estudiantes pueden desarrollar tanto tareas matemáticas vinculadas con el álgebra temprana, desde las primeras edades, como aprender las nociones algebraicas elementales (e.g. CARPENTER; FRANKE; LEVI, 2003; MOLINA, 2011; RADFORD, 2010, 2011), tales como reconocer patrones repetitivos, describir situaciones de igualdad y desigualdad, entre otras. En este sentido, el análisis de los libros de texto permite aproximarnos a esta realidad, puesto que este recurso didáctico influye, considerablemente, en el proceso de instrucción en el aula (EVEN; OLSHER, 2014), al constituirse en un elemento de apoyo para el profesorado en la preparación de la clase.

Con base en lo anterior, Demosthenous y Stylianides (2014) proponen un marco analítico para determinar las tareas matemáticas relacionadas con el álgebra temprana en los libros de texto de la Educación Primaria. En este marco se consideran tres categorías de tareas, que aportan una mirada amplia y general hacia las tareas matemáticas sobre álgebra temprana en los libros de texto:

a) Tareas de relaciones aritméticas situadas (RAS), centradas en la estructura de la aritmética atendiendo al comportamiento de las operaciones aritméticas y propiedades como objetos matemáticos y el por qué funcionan de esta manera. Estas tareas podrían involucrar a los estudiantes en la generalización de estas relaciones. En la literatura, esta categoría de tareas se conoce como aritmética generalizada (CARPENTER; FRANKE; LEVI, 2003; KAPUT, 2008). Por ejemplo: una tarea que pide a los estudiantes formar una expresión general para la propiedad conmutativa de la adición.

b) Tareas de relaciones basadas en regla $(R B R)$, centradas en las relaciones de un conjunto de datos o entre conjuntos de datos. Estas tareas podrían involucrar a los estudiantes en la formación de una regla que se aplique a todos los elementos de los conjuntos de datos comprobando las reglas plausibles, extendiendo una regla para casos cercanos y lejanos, y generalizando una regla. Así también, la tarea podría ofrecer oportunidades para trabajar con representaciones equivalentes de la misma regla (por ejemplo, verbales y algebraicas). Por ejemplo: una tarea que pide a los estudiantes que generalicen verbalmente la regla funcional de un patrón geométrico creciente. Esta categoría de tareas se relaciona con el estudio de los patrones, las funciones, el cambio (cambios cualitativos y cuantitativos, cambios que experimenta una variable, tasas de cambio contantes o variables) y la variación (KAPUT, 
2008; NCTM, 2000).

c) Tareas de relaciones conocidas-desconocidas ( $R C D)$, vinculadas con las relaciones entre cantidades y, números conocidos y desconocidos, y tratan a las incógnitas como objetos en lugar de como procesos. Por ejemplo, un problema como sigue: una granja tiene pollos y conejos. Contamos las cabezas y encontramos 27. Contamos los pies y encontramos 78. ¿Cuántos son los pollos y cuántos los conejos? Esta categoría de tareas se fundamenta en la descripción del álgebra como un conjunto de lenguajes de modelización (KAPUT, 2008) y el enfoque de la resolución de problemas sobre la introducción al álgebra (BEDNARZ; KIERAN; LEE, 1996).

Algunos trabajos que analizan series de libros de texto, con base en estas categorías, aportan evidencia acerca de los tipos de tareas algebraicas que estos emplazan en diversos niveles educativos. Demosthenous y Stylianides (2014), por ejemplo, analizaron una serie de libros de texto de Educación Primaria para cuarto, quinto y sexto grado (9-12 años) y obtuvieron que, de las 2814 tareas que contenían, el 10,7\% estaban relacionadas con el álgebra, entre las que prevalecen las tareas matemáticas basadas en reglas $(42,4 \%)$ y las tareas de relaciones conocidas-desconocidas $(45,7 \%)$.

Salazar, Cabañas-Sánchez y Navarro (2016) analizaron las tareas matemáticas relacionadas al álgebra temprana en los libros de texto de segundo y tercer grado de Educación Primaria (7-9 años). Los resultados muestran que, de las 140 tareas analizadas, la mayoría se ubican en la categoría de relaciones aritméticas situadas (79,3\%), seguidas de las tareas de relaciones basadas en regla $(15,7 \%)$ y un porcentaje menor corresponden a tareas de relaciones conocidas-desconocidas (5\%). En general, se evidencia que las tareas matemáticas correspondientes a este bloque de contenido tienen escasa presencia en los primeros grados de Educación Primaria.

Asimismo, Cabañas-Sánchez, Salazar y Nolasco-Hesiquio (2017) analizaron las tareas matemáticas que potencian el desarrollo del pensamiento algebraico en primer grado de Educación Primaria (6-7 años). Se analizaron un total de 125 tareas, de las cuales 68 corresponden al estudio del álgebra temprana. En su mayoría, las tareas se ubican en las categorías de relaciones aritméticas situadas (44\%) y relaciones basadas en regla (43\%), una cantidad menor de tareas corresponden a las de tipo de relaciones conocidas-desconocidas $(13 \%)$.

La multidimensionalidad de tareas que se describen en estos estudios responde a la propuesta de cambio curricular que promueve el álgebra temprana. Como se ha indicado en la introducción, diversos currículos de matemáticas de Educación Primaria han empezado a 
incorporar, progresivamente, estos contenidos. A modo de ejemplo, en la Figura 1 se muestra la presencia del álgebra en los estándares para las matemáticas propuestos por el National Council of Teachers of Mathematics, y su progresión a lo largo de las diferentes etapas (NCTM, 2000).

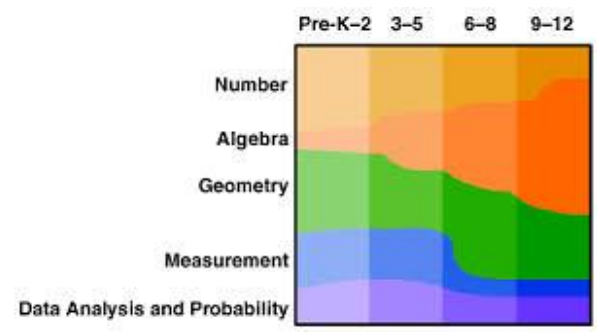

Figura 1 - Diferente atención de los Estándares de contenido Fuente: NCTM (2000, p. 30)

Como se aprecia en la Figura 1, la presencia del álgebra va aumentando progresivamente desde finales de la etapa Pre-K-2 (3 a 8 años) y durante toda la etapa 3-5 (9 a 12 años) y, con ello, se pretende que el alumnado de Educación Primaria pueda construir, gradualmente, una base sólida de conocimientos para el aprendizaje del álgebra a medida que aumenta el nivel y, de este modo, alcanzar con éxito el estudio de matemáticas más avanzadas (BLANTON et al., 2015). En lo que se refiere a los primeros niveles, explicitan los siguientes contenidos (Figura 2).

\begin{tabular}{|c|c|c|}
\hline $\begin{array}{l}\text { ESTÁNDAR } \\
\text { Los programas de enseñanza de todas las etapas } \\
\text { deberían capacitar a todos los estudiantes para: }\end{array}$ & $\begin{array}{l}\text { Etapa Pre-K-2 } \\
\text { Expectativas } \\
\text { En la etapa Pre-K-2, todos los estudiantes deberian: }\end{array}$ & $\begin{array}{l}\text { Etapa } 3-5 \\
\text { Expectativas } \\
\text { En la etapa 3-5, todos los estudiantes deberian: }\end{array}$ \\
\hline $\begin{array}{l}\text { Comprender patrones, relaciones y funcio- } \\
\text { nes }\end{array}$ & $\begin{array}{l}\text { - seleccionar, clasificar y ordenar objetos por el tamaño, la cantidad } \\
\text { y otras propiedades; } \\
\text { reconocer, describir y ampliar patrones tales como secuencias de } \\
\text { sonidos y formas o sencillos patrones numéricos, y pasar de una } \\
\text { representación a otra; } \\
\text { analizar cómo se generan patrones de repetición y de creci- } \\
\text { miento. }\end{array}$ & $\begin{array}{l}\text { - describir y extender patrones geométricos y numéricos y hacer } \\
\text { generalizaciones acerca de ellos; } \\
\text { representar y analizar patrones y funciones, verbalmente y me- } \\
\text { diante tablas y gráficas. }\end{array}$ \\
\hline $\begin{array}{l}\text { Representar y analizar situaciones y estruc- } \\
\text { turas matemáticas utilizando simbolos alge- } \\
\text { braicos }\end{array}$ & $\begin{array}{l}\text { - ilustrar los principios generales y las propiedades de las opera- } \\
\text { ciones, como la conmutatividad, usando números; } \\
\text { - usar representaciones concretas, pictóricas y verbales para de- } \\
\text { sarrollar la comprensión de notaciones simboólicas inventadas y } \\
\text { convencionales; }\end{array}$ & $\begin{array}{l}\text { - identificar propiedades como la conmutatividad, la asociatividad y } \\
\text { la distributividad, y emplearlas en el cálculo con números natu- } \\
\text { rales; } \\
\text { - representar la idea de variable como cantidad desconocida, por } \\
\text { medio de una letra o un simbolo; } \\
\text { - expresar relaciones matemáticas mediante ecuaciones. }\end{array}$ \\
\hline $\begin{array}{l}\text { Usar modelos matemáticos para representar } \\
\text { y comprender relaciones cuantitativas }\end{array}$ & $\begin{array}{l}\text { - modelizar situaciones relativas a la adición y sustracción de } \\
\text { números naturales, utilizando objetos, dibujos y simbolos. }\end{array}$ & $\begin{array}{l}\text { modelizar situaciones de problema con objetos, y usar repre- } \\
\text { sentaciones como gráficas, tablas y ecuaciones para extraer con- } \\
\text { clusiones. }\end{array}$ \\
\hline Analizar el cambio en contextos diversos & $\begin{array}{l}\text { - describir cambios cualitativos, como "ser más alto"; } \\
\text { - describir cambios cuantitativos, como el aumento de estatura de } \\
\text { un alumno en dos pulgadas en un ano. }\end{array}$ & $\begin{array}{l}\text { - investigar de qué manera el cambio que experimenta una variable } \\
\text { se relaciona con el de una segunda variable; } \\
\text { - identificar y describir situaciones con tasas de cambio constantes } \\
\text { o variables, y compararlas. }\end{array}$ \\
\hline
\end{tabular}

Figura 2 - Estándares de álgebra para la Etapa Pre-K-2 (3-8 años) y la Etapa 3-5 (9-12 años) Fuente: NCTM (2000, p. 402)

A partir de la revisión de las orientaciones curriculares de Estados Unidos (CCSSM, 2010; NCTM, 2000) y de otros países que ponen en manifiesto el estudio del álgebra temprana desde los primeros años de escolaridad, como Australia (ACARA, 2015), Singapur 
(MINISTRY OF EDUCATION, REPUBLIC OF SINGAPORE, 2012, 2013) y Chile (MINEDUC, 2012, 2018), junto con las aportaciones sobre los conocimientos importantes de álgebra temprana planteados por diversos autores (ALSINA, 2019; CARPENTER; FRANKE; LEVI, 2003; KAPUT, 2000; MOLINA, 2011; MOLINA; CAÑADAS, 2018; RADFORD, 2010, 2011; SCHLIEMANN et al., 2003; entre otros), Pincheira y Alsina (2021) han realizado una primera caracterización de los conocimientos matemáticos que promueven el desarrollo del pensamiento algebraico temprano en Educación Infantil y Primaria.

En concreto, para la Educación Primaria establecen, inicialmente, cuatro categorías de conocimientos: a) comprensión de distintos tipos de relaciones y patrones: centrado en cómo se relacionan unas cantidades con otras, a partir de equivalencias, ordenaciones, desigualdades etc., establecer generalizaciones e identificar patrones y regularidades; b) uso de símbolos algebraicos y modelos para representar situaciones matemáticas: asociado a la notación simbólica a través de objetos, dibujos o lenguaje formal y, la modelización de situaciones del mundo real; c) comprensión del cambio: vinculado con el análisis de cambios cualitativos o cuantitativos en diversos contextos; d) uso de variables para determinar una constante o incógnita: referente a las condiciones que pueden tomar los valores de una variable como cantidad desconocida.

\section{Método}

De acuerdo con el objetivo de nuestro estudio, se ha utilizado una metodología cualitativa de carácter descriptivo (HERNÁNDEZ; FERNÁNDEZ; BAPTISTA, 2010), usando como técnica el análisis de contenido, que es "una técnica de investigación para hacer inferencias replicables y válidas a partir del texto (u otra materia significativa) a los contextos de su uso" (KRIPPENDORFF, 2013, p. 24). Para llevar a cabo el análisis de contenido, hemos adaptado ciertos elementos de la estructura propuesta por Cobo (2003) para el análisis de libros de texto, considerando las siguientes etapas:

1. Reconocer y seleccionar las unidades de análisis, constituidas por las secciones de los libros de texto que presentan tareas matemáticas para abordar conocimientos vinculados al álgebra temprana.

2. Determinar las categorías e indicadores de análisis, a partir de la articulación entre el marco analítico propuesto por Demosthenous y Stylianides (2014) y la caracterización del álgebra temprana de Pincheira y Alsina (2021).

3. Codificar las tareas matemáticas relacionadas con el estudio del álgebra 
temprana presentes en los libros de texto con base en estas categorías e indicadores.

4. Sistematizar la información por medio de tablas estadísticas, cuya lectura facilite el análisis descriptivo y la obtención de conclusiones.

5. Evidenciar el análisis descriptivo, por medio de la selección de ejemplos de tareas matemáticas propuestas en los textos de acuerdo a cada una de las categorías de análisis establecidas.

\subsection{Muestra}

La muestra está constituida por una colección de ocho libros de texto chilenos (Cuadro 1) de Educación Primaria ( $1^{\circ}$ a $6^{\circ}$ grado) que se encuentran en concordancia con las orientaciones curriculares vigentes de matemáticas en Chile (MINEDUC, 2012). Dicha muestra se ha escogido de manera intencionada dado el impacto que posee, puesto que los libros de texto seleccionados corresponden a textos oficiales con los cuales se orienta la enseñanza de la matemática escolar en los establecimientos educacionales chilenos, tanto públicos como particulares subvencionados $(94,1 \%)$, siendo distribuidos de manera gratuita por el Ministerio de Educación.

\begin{tabular}{|c|c|c|c|}
\hline Código & $\begin{array}{c}\text { Título } \\
\end{array}$ & Autores & Editorial \\
\hline $\mathrm{T} 1$ & $\begin{array}{c}\text { Sumo Primero Tomo } 1 \\
1^{\circ} \text { Básico }\end{array}$ & Masami Isoda (2020) & Mineduc \\
\hline $\mathrm{T} 2$ & $\begin{array}{c}\text { Sumo Primero Tomo } 2 \\
1^{\circ} \text { Básico }\end{array}$ & Masami Isoda (2020) & Mineduc \\
\hline $\mathrm{T} 3$ & $\begin{array}{c}\text { Sumo Primero Tomo } 1 \\
2^{\circ} \text { Básico }\end{array}$ & Masami Isoda (2020) & Mineduc \\
\hline $\mathrm{T} 4$ & $\begin{array}{l}\text { Sumo Primero Tomo } 2 \\
2^{\circ} \text { Básico }\end{array}$ & Masami Isoda (2020) & Mineduc \\
\hline $\mathrm{T} 5$ & Matemática $3^{\circ}$ Básico & $\begin{array}{c}\text { Andrea Urra Vásquez, Carmen Córdoba } \\
\text { Hermosilla y Claudia Quezada Soto } \\
(2019)\end{array}$ & Santillana \\
\hline T6 & Matemática $4^{\circ}$ Básico & $\begin{array}{c}\text { Romina Rodriguez Rojel, Daniela } \\
\text { García Orellana, Patricia Romante } \\
\text { Flores y Arlette Verdejo Lagunas (2019) }\end{array}$ & SM \\
\hline $\mathrm{T} 7$ & Matemática $5^{\circ}$ Básico & $\begin{array}{c}\text { Fong Ho Kheong, Gan Kee Soon y } \\
\text { Chelvi Ramakrishnan (2019) }\end{array}$ & Santillana \\
\hline $\mathrm{T} 8$ & Matemática $6^{\circ}$ Básico & $\begin{array}{c}\text { Lesly Maldonado Rodríguez y Carlos } \\
\text { Castro Maldonado (2019) }\end{array}$ & Santillana \\
\hline
\end{tabular}

Cuadro 1 - Colección de libros de texto considerados para el análisis

Fuente: elaboración propia

A partir de la muestra, se han seleccionado las unidades de análisis que corresponden a las tareas matemáticas vinculadas al estudio del álgebra temprana. Cabe destacar que los libros de texto correspondientes a los niveles de $1^{\circ}$ Básico y $2^{\circ}$ Básico se subdividen en dos series de textos (Tomo 1 y 2), como se aprecia en el Cuadro 1. Para el desarrollo de la 
investigación, se han analizado ambas subseries de textos, de manera transversal al nivel educativo que corresponden, dado que uno es la continuación del otro.

\subsection{Categorías e indicadores de análisis}

Para llevar a cabo el estudio, como se ha indicado, las unidades de análisis se han examinado con base en las tres categorías de tareas relacionadas con el álgebra temprana en los libros de texto, planteadas por Demosthenous y Stylianides (2014): a) tareas de relaciones aritméticas situadas, b) tareas de relaciones basadas en reglas y, c) tareas de relaciones conocidas-desconocidas. Estas categorías se han articulado con las categorías de conocimientos que caracterizan el álgebra temprana en Educación Primaria propuestos por Pincheira y Alsina (2021), al reconocer relaciones o estructuras matemáticas asociadas al tipo de razonamiento que demanda el proceso de resolución de tareas sobre el pensamiento algebraico temprano:

- Las tareas de relaciones aritméticas situadas se conectan con la comprensión de distintos tipos de relaciones, la solución de este tipo de tareas se vincula, explícitamente, con la idea de equivalencia, y, de modo implícito, con la desigualdad (CABAÑAS-SÁNCHEZ; SALAZAR; NOLASCO-HESIQUIO, 2017). A su vez, en este tipo de tareas se establecen relaciones de orden y comparación entre cantidades.

- Las tareas de relaciones basadas en reglas promueven el reconocimiento de patrones en sucesiones y desarrollo del pensamiento funcional (CABAÑAS-SÁNCHEZ; SALAZAR; NOLASCO-HESIQUIO, 2017; DEMOSTHENOUS; STYLIANIDES, 2014). De este modo, dichas tareas se enmarcan en la comprensión de patrones y comprensión del cambio.

- Las tareas de relaciones conocidas-desconocidas se articulan con problemas de valor faltante y el lenguaje de modelización (DEMOSTHENOUS; STYLIANIDES, 2014), vinculándose directamente con el uso de símbolos algebraicos y modelos matemáticos para representar situaciones y, uso de variables para determinar una constante o incógnita.

La articulación de ambos enfoques ha permitido definir un conjunto de indicadores para establecer las relaciones de las tareas matemáticas asociadas al estudio del álgebra temprana, y de esta forma, analizar de manera más precisa los conocimientos matemáticos que se deben movilizar en el proceso de resolución de tareas que promueven el pensamiento algebraico temprano en los libros de texto de Educación Primaria. En el marco de esta articulación (Cuadro 2), se ha subdividido en dos la categoría de conocimientos: $a$ ) Comprensión de distintos tipos de relaciones y patrones, ya que las relaciones mantienen 
vínculos con la categoría Tareas de relaciones aritméticas situadas (RAS), mientras que los patrones se vinculan con las Tareas de relaciones basadas en regla (RBR).

\begin{tabular}{|c|c|c|}
\hline $\begin{array}{c}\text { Categorías de } \\
\text { tareas }\end{array}$ & & Indicadores \\
\hline RAS & $\begin{array}{l}\text { Comprensión de } \\
\text { distintos tipos } \\
\text { de relaciones }\end{array}$ & $\begin{array}{l}\text { 1. Establecer relaciones de equivalencia entre dos cantidades. } \\
\text { 2. Establecer relaciones de igualdad o desigualdad entre cantidades. } \\
\text { 3. Establecer relaciones de comparación y/u orden entre cantidades. }\end{array}$ \\
\hline RBR & $\begin{array}{c}\text { Comprensión de } \\
\text { patrones }\end{array}$ & $\begin{array}{l}\text { 4. Crear secuencias o patrones. } \\
\text { 5. Completar elementos faltantes en secuencias numéricas o lógicas. } \\
\text { 6. Utilizar tablas de } 100 \text { para determinar secuencias y/o patrones } \\
\text { numéricos (crecientes, decrecientes) } \\
\text { 7. Establecer relaciones o reglas entre los números que componen } \\
\text { una tabla de valores. } \\
\text { 8. Identificar un patrón a partir de una secuencia numérica, } \\
\text { secuencia lógica o en relación a los valores de una tabla. } \\
\text { 9. Determinar los términos de una secuencia considerando el patrón } \\
\text { de formación. } \\
\text { 10. Determinar términos de una tabla de valores a partir de un patrón } \\
\text { o secuencia. }\end{array}$ \\
\hline & $\begin{array}{l}\text { Comprensión } \\
\text { del cambio. }\end{array}$ & $\begin{array}{l}\text { 11. Identificar y/o describir cambios cualitativos. } \\
\text { 12. Identificar y/o describir cambios cuantitativos. } \\
\text { 13. Analizar situaciones en las que se producen cambios y otras que } \\
\text { se mantienen constantes. }\end{array}$ \\
\hline \multirow[t]{2}{*}{$\mathrm{RCD}$} & $\begin{array}{l}\text { Uso de símbolos } \\
\text { algebraicos y } \\
\text { modelos } \\
\text { matemáticos } \\
\text { para representar } \\
\text { situaciones } \\
\text { matemáticas. }\end{array}$ & $\begin{array}{l}\text { 14. Representar una ecuación/inecuación por medio de balanza o } \\
\text { viceversa. } \\
\text { 15. Representar expresiones escritas en lenguaje natural con lenguaje } \\
\text { algebraico o viceversa. } \\
\text { 16. Utilizar expresiones algebraicas para establecer relaciones entre } \\
\text { números. } \\
\text { 17. Identificar regularidades en una situación y utilizar símbolos } \\
\text { algebraicos para expresarla. } \\
\text { 18. Plantear y/o resolver ecuaciones de primer grado con una } \\
\text { incógnita. } \\
\text { 19. Plantear y/o resolver inecuaciones. }\end{array}$ \\
\hline & $\begin{array}{l}\text { Uso de variables } \\
\text { para determinar } \\
\text { una constante o } \\
\text { incógnita. }\end{array}$ & $\begin{array}{l}\text { 20. Evaluar expresiones de una variable. } \\
\text { 21. Analizar las condiciones de los valores que puede tomar una } \\
\text { variable. } \\
\text { 22. Determinar los valores de la variable que permiten mantener y/o } \\
\text { dar solución a una ecuación o inecuación. }\end{array}$ \\
\hline
\end{tabular}

Cuadro 2 - Categorías e indicadores utilizados en el proceso de codificación Fuente: elaboración propia

La codificación de los datos se ha llevado a cabo en tres fases: 1) la clasificación de las tareas matemáticas sobre álgebra temprana con base en las categorías propuestas por Demosthenous y Stylianides (2014); 2) la delimitación de las tareas clasificadas a partir de la caracterización del álgebra temprana en Educación Primaria propuesta por Pincheira y Alsina (2021); 3) y finalmente, el uso de los indicadores correspondientes a cada tipo de tarea matemática de acuerdo con la asignación de puntuaciones en caso de presencia (1 punto) o ausencia ( 0 puntos) en las lecciones vinculada al estudio del álgebra temprana. Para establecer la codificación de los datos se han realizado revisiones sucesivas de los textos escolares, de modo cíclico e inductivo. 


\section{Resultados}

Considerando nuestro objetivo de estudio, se describen los datos obtenidos a partir de la articulación del marco analítico propuesto por Demosthenous y Stylianides (2014) y la caracterización de los conocimientos de álgebra temprana para la Educación Primaria propuestos por Pincheira y Alsina (2021).

\subsection{Distribución de las tareas matemáticas propuestas en los libros de texto relacionadas con el estudio del álgebra temprana}

Para seleccionar las tareas matemáticas vinculadas con el estudio del álgebra temprana, en primer lugar, se ha partido de las lecciones destinadas al desarrollo de este bloque de contenido declaradas en los libros de texto. Dado que los libros de texto de $1^{\circ}$ y $2^{\circ}$ de Educación Primaria no presentan lecciones específicas para indagar en el estudio del álgebra temprana, se han seleccionado todas las tareas matemáticas que se vinculan con su enseñanza.

Por otra parte, en la selección se han considerado las tareas matemáticas sobre álgebra temprana propuestas en todas las secciones de los libros de texto: evaluación inicial, desarrollo del contenido, evaluación de proceso, evaluación final.

La distribución de las tareas matemáticas de acuerdo con cada nivel educativo se aprecia en la Tabla 1.

Tabla 1 - Distribución de las tareas matemáticas analizadas

\begin{tabular}{ccc}
\hline Nivel Educativo & Frecuencia & Porcentaje \\
\hline $1^{\text {o }}$ Educación Primaria & 107 & 29 \\
$2^{\text {o }}$ Educación Primaria & 41 & 11 \\
$3^{\text {o }}$ Educación Primaria & 45 & 12 \\
$4^{\circ}$ Educación Primaria & 49 & 13 \\
$5^{\circ}$ Educación Primaria & 15 & 4 \\
$6^{\circ}$ Educación Primaria & 116 & 31 \\
\hline \multicolumn{2}{c}{ Fuente: elaboración propia }
\end{tabular}

Se han analizado un total de 373 tareas matemáticas vinculadas al estudio del álgebra temprana, observando que su enseñanza se encuentra presente en los libros de texto de todos los niveles de Educación Primaria. Asimismo, se evidencia una concentración mayor de tareas en el primer y último nivel de Educación Primaria, mientras que en $5^{\circ}$ Educación Primaria la presencia de la actividad algebraica temprana en los libros de texto es considerablemente menor, con un $4 \%$. 


\subsection{Análisis de las categorías de tareas y de los conocimientos matemáticos que caracterizan la enseñanza del álgebra temprana}

En la Tabla 2 se muestran los datos correspondientes a la articulación de las categorías de las tareas relacionadas con el álgebra temprana que plantean Demosthenous y Stylianides (2014) y las categorías de conocimientos que caracterizan la enseñanza del álgebra temprana en Educación Primaria (PINCHEIRA; ALSINA, 2021).

Tabla 2 - Distribución por porcentaje de las categorías de tareas relacionadas con el álgebra temprana y los conocimientos que caracterizan el álgebra temprana en Educación Primaria

\begin{tabular}{|c|c|c|c|c|c|c|c|c|}
\hline $\begin{array}{l}\text { Categorías } \\
\text { de tareas }\end{array}$ & Caracterización & $\begin{array}{c}1^{\circ} \text { E.P } \\
(n=107)\end{array}$ & $\begin{array}{l}2^{\circ} \text { E.P } \\
(n=41)\end{array}$ & $\begin{array}{l}3^{\circ} \text { E.P } \\
(n=45)\end{array}$ & $\begin{array}{l}4^{\circ} \text { E.P } \\
(n=49)\end{array}$ & $\begin{array}{l}5^{\circ} \text { E.P } \\
(n=15) \\
\end{array}$ & $\begin{array}{c}6^{0} \text { E.P } \\
(n=116)\end{array}$ & $\begin{array}{c}\text { Total } \\
(\mathrm{n}=373)\end{array}$ \\
\hline RAS & $\begin{array}{l}\text { Comprensión de } \\
\text { distintos tipos de } \\
\text { relaciones }\end{array}$ & 38,3 & 46,3 & 15,6 & 6,1 & 0 & 7,8 & 21,2 \\
\hline \multirow{2}{*}{ RBR } & $\begin{array}{c}\text { Comprensión de } \\
\text { patrones }\end{array}$ & 29 & 19,6 & 53,4 & 24,5 & 100 & 24,1 & 31,6 \\
\hline & $\begin{array}{l}\text { Comprensión del } \\
\text { cambio }\end{array}$ & 32,7 & 34,1 & 0 & 0 & 0 & 0 & 13,1 \\
\hline \multirow[t]{2}{*}{$\mathrm{RCD}$} & $\begin{array}{l}\text { Uso de símbolos } \\
\text { algebraicos y } \\
\text { modelos } \\
\text { matemáticos para } \\
\text { representar } \\
\text { situaciones } \\
\end{array}$ & 0 & 0 & 31 & 61,2 & 0 & 50,9 & 27,6 \\
\hline & $\begin{array}{l}\text { Uso de variables } \\
\text { para determinar } \\
\text { una constante o } \\
\text { incógnita }\end{array}$ & 0 & 0 & 0 & 8,2 & 0 & 17,2 & 6,5 \\
\hline
\end{tabular}

Fuente: elaboración propia

A nivel general, los datos de la Tabla 2 muestran una mayor concentración de tareas de relaciones basadas en regla (RBR), con un $44,7 \%$, destacando las tareas vinculadas a la comprensión de patrones $(31,6 \%)$. Siguen las tareas de relaciones conocidas-desconocidas (RCD), con un $34,1 \%$, donde predominan las tareas relacionadas con el uso de símbolos algebraicos y modelos matemáticos para representar situaciones $(27,6 \%)$. Por último, se observa una menor presencia de las tareas de relaciones aritméticas situadas (RAS), con un $21,2 \%$.

Así también, se observa que en los niveles de $1^{\circ}, 2^{\circ}, 3^{\circ}$ y $5^{\circ}$ Educación Primaria, las tareas RBR predominan sobre las tareas de RAS y tareas de RCD, con un 61,7\%, 53,7\%, $53,4 \%$ y $100 \%$, respectivamente. Cabe destacar que este tipo de tareas se encuentra presente en todos los libros de texto de Educación Primaria analizados.

En lo que respecta a las tareas de $\mathrm{RCD}$, comienzan a desarrollarse a partir de $3^{\circ}$ Educación Primaria, a excepción $5^{\circ}$ de Educación Primaria, alcanzando una mayor presencia 
en los niveles $4^{\circ}$ y $6^{\circ}$ Educación Primaria, con un $69,4 \%$ y 68,1\% respectivamente.

Por otro lado, en relación con las tareas de RAS se encuentran presentes en casi la totalidad de los libros de texto, exceptuando el $5^{\circ}$ Educación Primaria.

Si nos situamos, de manera más específica, desde la perspectiva de los indicadores que se han definido para establecer las relaciones de las tareas matemáticas asociadas al estudio del álgebra temprana (Cuadro 2), en la Tabla 3 es posible observar los indicadores que predominan respecto de las tareas matemáticas analizadas en cada nivel educativo de $1^{\circ}$ a $6^{\circ}$ de Educación Primaria. Cabe destacar que una determinada tarea matemática puede requerir atender a uno o más de los indicadores propuestos, razón por la cual a una tarea puede no corresponderle un solo indicador.

Tabla 3 - Distribución por porcentaje de los indicadores que caracterizan las tareas matemáticas de álgebra temprana en Educación Primaria en relación al total de tareas analizadas para cada nivel educativo

\begin{tabular}{|c|c|c|c|c|c|c|c|c|}
\hline $\begin{array}{l}\text { Categorías } \\
\text { de tareas }\end{array}$ & Caracterización & Indicadores & $\begin{array}{l}1^{\circ} \\
\text { E.P }\end{array}$ & $\begin{array}{l}2^{\mathbf{o}} \\
\text { E.P }\end{array}$ & $\begin{array}{l}\mathbf{3}^{\mathbf{o}} \\
\text { E.P }\end{array}$ & $\begin{array}{l}4^{\circ} \\
\text { E.P }\end{array}$ & $\begin{array}{l}5^{\circ} \\
\text { E.P.P }\end{array}$ & $\begin{array}{l}6^{\circ} \\
\text { E.P }\end{array}$ \\
\hline \multirow{3}{*}{ RAS } & Comprensión de & 1 & 5,6 & 0 & 4,4 & 6,1 & 0 & 0 \\
\hline & distintos tipos de & 2 & 17,8 & 26,8 & 6,7 & 0 & 0 & 3,4 \\
\hline & relaciones & 3 & 15 & 19,5 & 4,4 & 0 & 0 & 0 \\
\hline \multirow{10}{*}{ RBR } & \multirow{7}{*}{$\begin{array}{l}\text { Comprensión de } \\
\text { patrones }\end{array}$} & 4 & 1,9 & 2,4 & 4,4 & 0 & 0 & 0,9 \\
\hline & & 5 & 26,2 & 14,6 & 11,1 & 0 & 13,3 & 3,4 \\
\hline & & 6 & 0 & 0 & 11,1 & 2 & 0 & 0 \\
\hline & & 7 & 0 & 2,4 & 2,2 & 10,2 & 13,3 & 13,8 \\
\hline & & 8 & 5,6 & 0 & 22,2 & 20,4 & 33,3 & 9,5 \\
\hline & & 9 & 0 & 0 & 6,7 & 4,1 & 40 & 5,2 \\
\hline & & 10 & 0 & 0 & 0 & 12,2 & 26,7 & 10,3 \\
\hline & \multirow{3}{*}{$\begin{array}{l}\text { Comprensión } \\
\text { del cambio }\end{array}$} & 11 & 0 & 0 & 0 & 0 & 0 & 0 \\
\hline & & 12 & 32,7 & 34,1 & 0 & 0 & 0 & 0 \\
\hline & & 13 & 0 & 0 & 0 & 0 & 0 & 0 \\
\hline \multirow{9}{*}{$\mathrm{RCD}$} & Uso de símbolos & 14 & 0 & 0 & 4,4 & 12,2 & 0 & 1,7 \\
\hline & algebraicos y & 15 & 0 & 0 & 2,4 & 6,1 & 0 & 13,8 \\
\hline & modelos & 16 & 0 & 0 & 4,4 & 0 & 0 & 10,3 \\
\hline & matemáticos & 17 & 0 & 0 & 0 & 0 & 0 & 12,1 \\
\hline & para representar & 18 & 0 & 0 & 20 & 26,5 & 0 & 18,1 \\
\hline & $\begin{array}{l}\text { situaciones } \\
\text { matemáticas }\end{array}$ & 19 & 0 & 0 & 0 & 18,3 & 0 & 0 \\
\hline & Uso de variables & 20 & 0 & 0 & 0 & 0 & 0 & 13,8 \\
\hline & para determinar & 21 & 0 & 0 & 0 & 8,1 & 0 & 6,3 \\
\hline & $\begin{array}{l}\text { una constante o } \\
\text { incógnita }\end{array}$ & 22 & 0 & 0 & 0 & 10,2 & 0 & 0 \\
\hline
\end{tabular}

Fuente: elaboración propia

En $1^{\circ}$ de Educación Primaria, los libros de texto proporcionan mayoritariamente tareas matemáticas que promueven la comprensión de distintos tipos de relaciones $(38,3 \%)$, sobresaliendo con un 17,8\% el indicador 2, que tiene relación con establecer relaciones de igualdad o desigualdad entre cantidades. Un ejemplo de este tipo de tarea se muestra en la Figura 3, puesto que, para responder de manera correcta, los estudiantes deben agregar los elementos faltantes para equilibrar la balanza y establecer la igualdad entre las cantidades. 
2 ¿Por qué no están equilibradas?

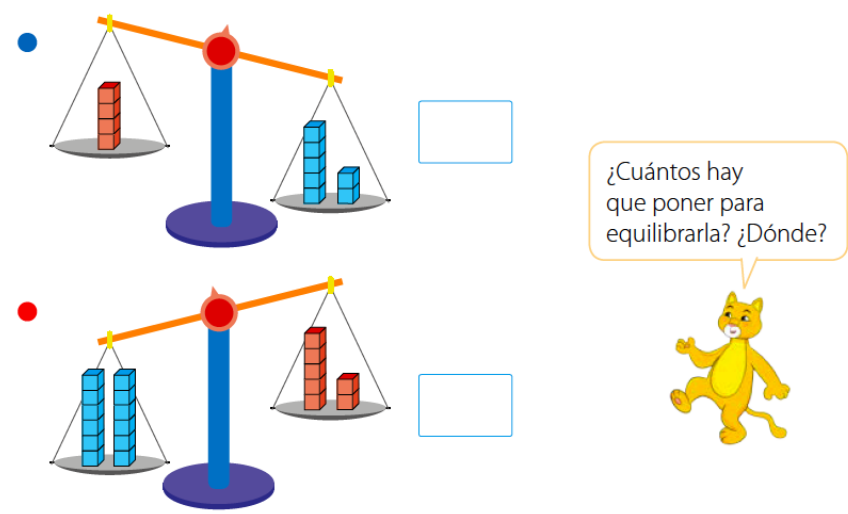

Figura 3 - Tarea de comprensión de distintos tipos de relaciones, indicador 2 Fuente: T2 (2020, p. 47)

$\mathrm{Al}$ analizar las tareas matemáticas propuestas en los libros de texto de $2^{\circ}$ Educación Primaria predominan las tareas matemáticas que involucran la comprensión de distintos tipos de relaciones $(46,3 \%)$, destacando el indicador 2, referido a establecer relaciones de igualdad o desigualdad entre cantidades (26,8\%). En la Figura 4 se muestra un ejemplo de este tipo de tarea.

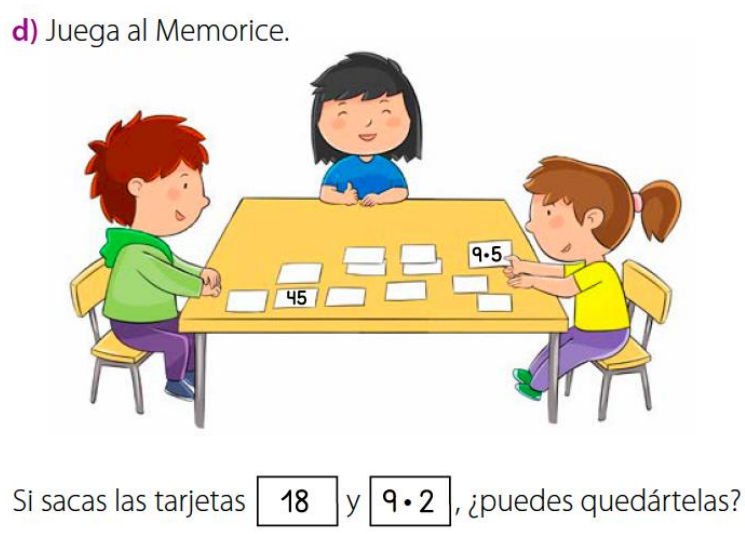

Figura 4 - Tarea de comprensión de distintos tipos de relaciones, indicador 2 Fuente: T4 (2020, p. 42)

Los estudiantes, para dar solución al problema, requieren establecer relaciones entre las cantidades que intervienen, en este caso la tarea demanda establecer una relación de igualdad entre los resultados de ambas tarjetas.

Por otra parte, en relación al libro de texto de $3^{\circ}$ Educación Primaria, destacan las tareas que requieren de la comprensión de patrones para su resolución $(53,4 \%)$, prevalece el indicador $8(22,2 \%)$, vinculado con la identificación de un patrón a partir de una secuencia numérica, secuencia lógica o en relación con los valores de una tabla. Un ejemplo de tarea que involucra la comprensión de patrones se presenta en la Figura 5: 
Observa los números que se muestran en los recuadros pintados en la tabla y luego responde.

\begin{tabular}{|r|r|r|r|r|r|r|r|r|r|}
\hline 1 & 2 & 3 & 4 & 5 & 6 & 7 & 8 & 9 & 10 \\
\hline 11 & 12 & 13 & 14 & 15 & 16 & 17 & 18 & 19 & 20 \\
\hline 21 & 22 & 23 & 24 & 25 & 26 & 27 & 28 & 29 & 30 \\
\hline
\end{tabular}

a. ¿Qué patrón observas en el dígito de las unidades y en el dígito de las decenas de los números contenidos en los recuadros pintados?

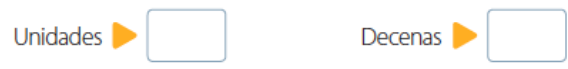

Figura 5 - Tarea de comprensión de patrones, indicador 8 Fuente: T5 (2019, p. 183)

Para dar solución a este tipo de tarea, los estudiantes deben determinar el patrón numérico a partir de los valores que muestra la tabla. Descubrir el patrón requiere observar los números de manera diagonal, identificando el sentido de la secuencia, ya sea creciente o decreciente.

En el caso del libro de texto de $4^{\circ}$ Educación Primaria, predominan las tareas que involucran el uso de símbolos algebraicos y modelos matemáticos para representar situaciones matemáticas $(61,2 \%)$, es el indicador 18 el que presenta una mayor presencia $(26,5 \%)$. Este indicador tiene relación con plantear y resolver ecuaciones de primer grado con una incógnita. Un ejemplo de este tipo de tarea es el siguiente (Figura 6):

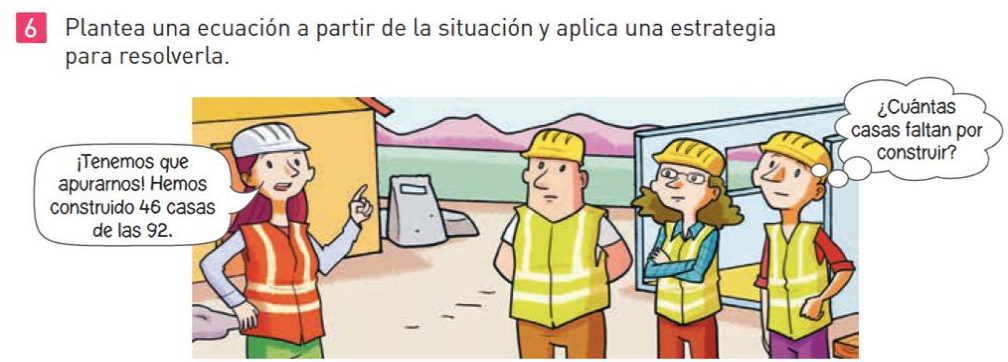

Figura 6 - Tarea de uso de símbolos algebraicos y modelos matemáticos para representar situaciones matemáticas, indicador 18

Fuente: T6 (2019, p. 105)

En la Figura 6, los estudiantes deben modelar una situación cotidiana mediante una ecuación. A su vez, el desarrollo de esta tarea requiere determinar el valor de la incógnita apoyándose en diversas estrategias, ya sea utilizando una balanza equilibrada descomponiendo los números involucrados o aplicando propiedades numéricas, determinando la operación inversa.

En cuanto al libro de texto de $5^{\circ}$ Educación Primaria, sólo promueve tareas matemáticas que requieren de la comprensión de patrones para su resolución. Destaca el indicador 9, que se focaliza en determinar los términos de una secuencia considerando el patrón de formación, con una presencia del 40\%. La Figura 7 ejemplifica este tipo de tarea. 
Escribe los 5 primeros términos de cada secuencia considerando la información dada.

a. El primer término es 45 y el patrón de formación es multiplicar por 10.

b. El primer término es 729 y el patrón de formación es dividir por 3.

Figura 7 - Tarea de comprensión de patrones, indicador 9 Fuente: T7 (2019, p. 89)

La solución de esta tarea requiere que los estudiantes utilicen el patrón de formación otorgado y lo apliquen en la secuencia numérica, de tal modo que esto les permita determinar términos en la secuencia y hacer predicciones.

Finalmente, el libro de texto de $6^{\circ}$ de Educación Primaria promueve mayoritariamente las tareas que suponen el uso de símbolos algebraicos y modelos matemáticos para representar situaciones matemáticas $(50,9 \%)$, logra una mayor presencia $(18,1 \%)$ el indicador 18 , orientado a plantear y resolver ecuaciones de primer grado con una incógnita. En la Figura 8 se expone un ejemplo de este tipo de tarea.

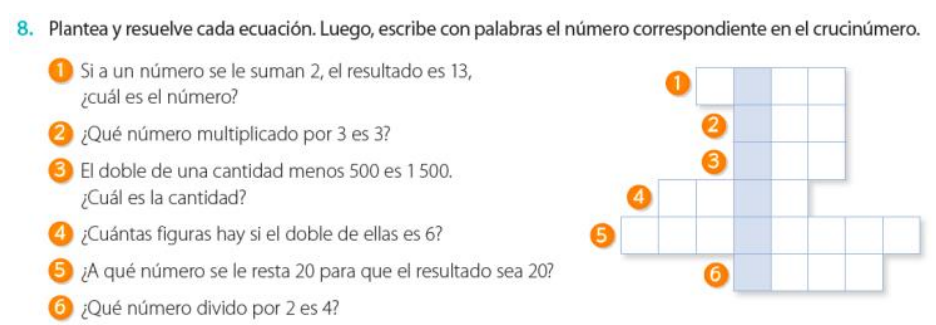

Figura 8 - Tarea de uso de símbolos algebraicos y modelos matemáticos para representar situaciones matemáticas, indicador 18 Fuente: T8 (2019, p. 129)

Los estudiantes, para dar solución a este tipo de tarea, deben aplicar ecuaciones de primer grado para modelar las diversas situaciones que se plantean, y posteriormente resolverlas. En este nivel educativo, se espera que los estudiantes evalúen el uso de diversas estrategias de resolución de ecuaciones y con ello las soluciones obtenidas según el contexto.

\section{Consideraciones finales}

En este estudio se ha presentado un análisis de las tareas matemáticas sobre álgebra temprana que proporcionan una colección de libros de texto de Educación Primaria (6 a 12 años) de amplia difusión en Chile. Dicho análisis se ha realizado a partir de la integración del marco analítico planteado por Demosthenous y Stylianides (2014) y la caracterización del álgebra temprana de Pincheira y Alsina (2021). Esta articulación ha permitido, por una parte, categorizar las tareas propuestas en los libros de texto sobre este bloque de contenido (DEMOSTHENOUS; STYLIANIDES, 2014): tareas de relaciones aritméticas situadas (RAS), tareas de relaciones basadas en regla (RBR) y tareas de relaciones conocidas y 
desconocidas (RCD); y, por otra parte, indagar en los conocimientos que se promueven en el desarrollo de dichas tareas matemáticas para conducir la enseñanza del álgebra temprana, de acuerdo con la caracterización del álgebra temprana (PINCHEIRA; ALSINA, 2021): comprensión de distintos tipos de relaciones y patrones, uso de símbolos algebraicos y modelos matemáticos para representar situaciones matemáticas, comprensión del cambio, uso de variables para determinar una constante o incógnita.

El análisis de las tareas matemáticas vinculadas al estudio del álgebra temprana en los libros de texto ha evidenciado la presencia y alcance de este bloque de contenidos en todos los niveles educativos de la Educación Primaria. No obstante, se observa un desajuste en la distribución de las tareas matemáticas, pues su incorporación no es progresiva durante esta etapa escolar como plantea, por ejemplo, el NCTM (2000). En $1^{\circ}$ Educación Primaria, por ejemplo, se observa una concentración mucho mayor de las tareas matemáticas que en los niveles posteriores, mientras que en $5^{\circ}$ Educación Primaria la incorporación de estas tareas es mínima.

De forma más concreta, los resultados obtenidos acerca del tipo de tareas muestran un predominio considerable de las tareas de relaciones basadas en regla $(44,7 \%)$, seguidas de las tareas de relaciones conocidas-desconocidas $(34,1 \%) \mathrm{y}$, por último, una presencia menor de las tareas de relaciones aritméticas situadas $(21,2 \%)$. Por un lado, estos resultados son innovadores en el contexto chileno, ya que hasta el momento no se tenían datos comparativos al respecto, y por otro, reportan semejanzas respecto de las tareas matemáticas sobre álgebra temprana analizadas en los libros de texto de otros países (e.g. CABAÑAS-SÁNCHEZ; SALAZAR; NOLASCO-HESIQUIO, 2017; DEMOSTHENOUS; STYLIANIDES, 2014; SALAZAR; CABAÑAS-SÁNCHEZ; NAVARRO, 2016). Así, se confirma que en los primeros niveles educativos prevalecen las tareas de relaciones aritméticas situadas, mientras que, a partir de los niveles intermedios predominan las tareas de relaciones basadas en regla, seguidas de las tareas de relaciones conocidas-desconocidas.

En lo que respecta a los conocimientos algebraicos que se movilizan en el proceso de resolución de las tareas matemáticas que proponen los libros de texto, se evidencia una fuerte presencia de las tareas que involucran la comprensión de distintos tipos de relaciones y patrones $(52,8 \%)$, seguidas de las tareas donde interviene el uso de símbolos algebraicos y modelos matemáticos para representar situaciones matemáticas $(27,6 \%)$, una menor presencia se observa en las tareas que requieren de la comprensión del cambio $(13,1 \%)$ para su resolución y uso de variables para determinar una constante o incógnita $(6,5 \%)$.

Estos resultados son relevantes, pues proporcionan evidencias acerca de como se está 
abordando la enseñanza del álgebra temprana en los libros de texto. Más concretamente, el análisis realizado ha permitido visualizar cuáles son los conocimientos que se profundizan en los diferentes niveles escolares para alcanzar el desarrollo del pensamiento algebraico, observando que su distribución no es homogénea.

Por otro lado, se evidencia una ausencia de tareas matemáticas que requieran identificar o describir cambios cualitativos y, analizar situaciones en las que se producen cambios y otras que se mantienen constantes. Para remediar esta situación, el profesorado debería ofrecer oportunidades de aprendizaje que permitieran abordar, en su totalidad, el conocimiento en torno a la comprensión del cambio.

A modo general, los resultados favorecen la reflexión e invitan a abrir la discusión acerca de ¿qué debe considerar la formación docente para promover una enseñanza idónea del álgebra temprana? y ¿cómo se deberían orientar las prácticas de enseñanza de este bloque de contenido?, pues es importante construir una base sólida de comprensión y manejo de experiencias acerca del álgebra temprana (NCTM, 2000), dado que esto un aspecto de gran interés para el desarrollo profesional del profesorado.

Bajo esta mirada, consideramos que es necesario proporcionar, tanto en la formación inicial como continua del profesorado, experiencias de formación que permitan profundizar en el análisis de tareas matemáticas que promuevan el desarrollo del pensamiento algebraico temprano, de modo tal, que se entreguen herramientas orientadoras para una adecuada selección e implementación de dichas tareas. En este sentido, es necesario que estas experiencias formativas también incorporen el análisis de las tareas planteadas en los libros de textos para seleccionar aquellas que favorezcan el desarrollo del pensamiento algebraico, o bien, enriquecerlas para que promuevan dicho pensamiento.

Asimismo, es recomendable que el profesorado favorezca la incorporación progresiva de las tareas algebraicas tempranas, a medida que avanzan los niveles escolares en Educación Primaria.

En consecuencia, conducir la enseñanza del álgebra temprana de manera efectiva, desde los primeros niveles educativos, requiere que el profesorado conozca e identifique las diversas categorías de tareas que se establecen para el tratamiento de este contenido, como también los conocimientos que caracterizan cada tarea. Esto último permitirá establecer relaciones y determinar en profundidad la actividad matemática que deben poner en juego los estudiantes para su resolución.

En cuanto a las limitaciones de la investigación, las tareas matemáticas analizadas no consideran, en su totalidad, el caso de la generalización de la aritmética, más específicamente 
el estudio sobre las propiedades de las operaciones.

Finalmente, la investigación permite abrir futuras líneas de indagación ampliando el estudio a otras colecciones de libros escolares. Así también, se hace necesario indagar en la demanda cognitiva de las tareas matemáticas propuestas para promover el aprendizaje del álgebra temprana en los libros de texto y en tratamiento que se les otorga a los contenidos vinculados con el álgebra temprana en los libros de texto de Educación Infantil.

\section{Agradecimientos}

Este trabajo fue apoyado por la Agencia Nacional de Investigación y Desarrollo del Gobierno de Chile (ANID) mediante una beca de doctorado en el extranjero, Folio $\mathrm{N}^{\circ}$ 72200447.

\section{Referencias}

ACARA. AUSTRALIAN CURRICULUM, ASSESSMENT AND REPORTING AUTHORITY. The Australian Curriculum: Mathematics. Australian curriculum. 2015. Disponible en: https://www.australiancurriculum.edu.au/f-10-curriculum/mathematics/. Acceso: 10 jun. 2020.

AKÉ, L. P.; GODINO, J. D. Análisis de tareas de un libro de texto de primaria desde la perspectiva de los niveles de algebrización. Revista Educación Matemática, Ciudad de México, v. 30, n. 2, p. 171201, 2018.

ALSINA, Á. Itinerarios didácticos para la enseñanza de las matemáticas (6-12 años). Barcelona: Graó, 2019.

BASTABLE, V.; SCHIFTER, D. Classroom stories: examples of elementary students engaged in Early Algebra. En: KAPUT, J.; CARRAHER, D. W.; BLANTON M. L. (Eds.). Algebra in the Early Grades. Mahwah: Lawrence Erlbaum Associates, 2007. p. 165-184.

BEDNARZ, N.; KIERAN, C.; LEE, L. Approaches to Algebra: Perspectives for Research and Teaching. En: BERNARZ, N.; KIERAN, C.; LEE, L. (Eds.). Approaches to algebra. Dordrecht: Springer, 1996. p. 3-12.

BLANTON, M.; KAPUT, J. J. Characterizing a classroom practice that promotes algebraic reasoning. Journal for Research in Mathematics Education, Virginia, v. 36, n. 5, p. 412-446, 2005.

BLANTON, M.; STEPHENS, A.; KNUTH, E.; MURPHY, A.; ISLER, I.; KIM, J. S. The Development of Children's Algebraic Thinking: The Impact of a Comprehensive Early Algebra Intervention in Third Grade. Journal for Research in Mathematics Education, Virginia, v. 46, n. 1, p. 39-87, ener. 2015.

CABAÑAS-SÁNCHEZ, G.; SALAZAR, V.; NOLASCO-HESIQUIO, H. Tareas que potencian el desarrollo del pensamiento algebraico temprano en libros de texto de matemáticas de primaria. En: AKÉ, L.; CUEVAS-ROMO, J. (Eds.). Pensamiento algebraico en México desde diferentes enfoques. San Luis Potosí: Universidad Autónoma de San Luis Potosí, 2017. p. 13-35. 
CAI, J.; KNUTH, E. Early algebraization: A Global dialogue from multiple perspectives. Berlin: Springer, 2011.

CAMPANARIO, J. M. ¿Qué puede hacer un profesor como tú o un alumno como el tuyo con un libro de texto como éste? Una relación de actividades poco convencionales. Enseñanza de las Ciencias, Barcelona, v. 19, n. 3, p. 351-364, 2001.

CARPENTER, T. P.; FRANKE, M. L.; LEVI, L. Thinking mathematically: Integrating arithmetic y algebra in elementary school. Portsmouth: Heinemann, 2003.

CARRAHER, D. W.; SCHLIEMANN, A. D. Early algebra and algebraic reasoning. En: LESTER, F. $\mathrm{K}$. (Ed.). Second handbook of research on mathematics teaching and learning. Reston: NCTM e IAP, 2007. p. 669-705.

COBO, B. Significado de las medidas de posición central para los estudiantes de secundaria. 2003. 303f. Tesis (Doctorado en Didáctica de las Matemáticas) - Universidad de Granada, Granada, 2003.

CCSSM. COMMON CORE STATE STANDARS FOR MATHEMATICS. Common Core State Standards Initiative. Washington: National Governors Association for Best Practices and the Council of Chief State School Officers, 2010. Disponible en: http://www.corestandards.org/wpcontent/uploads/Math_Standards1.pdf._Acceso: 15 jun. 2020.

DEMOSTHENOUS, E.; STYLIANIDES, A. Algebra-Related Tasks in Primary School Textbooks. En: NICOL, C.; LILJEDAHL, P.; OESTERLE, S.; ALLAN, D. (Eds.). Proceedings of the Joint Meeting of PME 38 and PME-NA 36. v. 2. Vancouver: PME, 2014. p. 369-376.

EVEN, R.; OLSHER, S. Teachers as participants in textbook development: The Integrated Mathematics Wiki-book Project. En: LI, Y.; LAPPAN, G. (Eds.). Mathematics Curriculum in School Education. New York: Springer, 2014. p. 333-350.

HERNÁNDEZ, R.; FERNÁNDEZ, C; BAPTISTA, P. Metodología de la investigación. 5. ed. Ciudad de México: McGraw-Hill Interamericana, 2010.

KAPUT, J. Transforming algebra from an engine of inequity to an engine of mathematical power by "algebrafying" the K-12 curriculum. Dartmouth: National Center for Improving Student Learning and Achievement in Mathematics and Science, 2000.

KAPUT, J. What is algebra? What is algebraic reasoning? En: KAPUT, J.; CARRAHER, D. W.; BLANTON, M. L. (Eds.). Algebra in the early grades. New York: Lawrence Erlbaum Associates \& NCTM, 2008. p. 5-17.

KRIPPENDORFF, K. Content Analysis: An Introduction to Its Methodology. 3. ed. Thousand Oaks: Sage Publications, 2013.

MINEDUC. Bases Curriculares 2012: Educación Básica Matemática. Santiago de Chile: Unidad de Curriculum y Evaluación, 2012.

MINEDUC. Bases Curriculares 2018: Educación Parvularia. Santiago de Chile: Unidad de Curriculum y Evaluación, 2018.

MINISTRY OF EDUCATION, REPUBLIC OF SINGAPORE. Mathematics Syllabus: Primary on to six. Singapore: Curriculum Planning and Development Division, 2012. 
MINISTRY OF EDUCATION, REPUBLIC OF SINGAPORE. Nurturing Early Learners: A Curriculum for Kindergartens in Singapore. Singapore: Ministry of Education, 2013.

MOLINA, M. Integración del pensamiento algebraico en la educación básica. Un experimento de enseñanza con alumnos de 8-9 años. En: MARTINHO, M. H.; FERREIRA, R. A. T.; PONTE, J. P. (Eds.). Ensino e Aprendizagem da Álgebra: Actas do Encontro de Investigacao em Educacao Matemática. Póvoa do Varzim: EIEM, 2011. p. 27-51.

MOLINA, M. Una propuesta de cambio curricular: integración del pensamiento algebraico en educación primaria. PNA, Granada, v. 3, n. 3, p.135-156, 2009.

MOLINA, M.; CAÑADAS, M. La noción de estructura en early algebra. En: FLORES, P.; LUPIÁÑEZ, J. L.; SEGOVIA, I. (Eds.). Enseñar matemáticas: Homenaje a los profesores Francisco Fernández y Francisco Ruiz. Granada: Atrio, 2018. p. 129-141.

NCTM. Principles and Standards for School Mathematics. Reston: The National Council of Teachers of Mathematics, 2000.

PINCHEIRA, N.; ALSINA, Á. Hacia una caracterización del álgebra temprana a partir del análisis de los currículos contemporáneos de Educación Infantil y Primaria. Revista Educación Matemática, Ciudad de México, v. 33, n. 1, p. 153-180, 2021.

PORTER, A. Measuring the content of instruction: Uses in research and practice. Educational Researcher, Washington, v. 31, n. 7, p. 3-14, oct. 2002.

RADFORD, L. Elementary forms of algebraic thinking in young students. En: PINTO, M. F.; KAWASAKI, T. F. (Eds.). Proceedings of the 34th Conference of the International Group for the Psychology of Mathematics Education. 4. ed. Belo Horizonte: PME, 2010. p. 73-80.

RADFORD, L. Embodiment, perception and symbols in the development of early algebraic thinking. En: UBUZ, B. (Ed.). Proceedings of the $3^{\text {th }}$ Conference of the International Group for the Psychology of Mathematics Education Developing Mathematical Thinking. 4. ed. Ankara: PME, 2011. p. 17-24.

RAMÍREZ, T. El texto escolar: una línea de investigación en educación. Revista de Pedagogía, Caracas, v. 24, n. 70, p. 273-292, 2003.

REMILLARD, J. T. Can curriculum materials support teachers' learning? Two fourth-grade teachers' use of a new mathematics text. The Elementary School Journal, Chicago, v. 100, n. 4, p. 331-350, 2000 .

SALAZAR, V.; CABAÑAS-SÁNCHEZ, G.; NAVARRO, C. Tareas relacionadas con el álgebra temprana en los libros de texto de matemáticas de primaria. Investigación e Innovación en Matemática Educativa, Chilpancingo de los Bravo, v. 2, n. 1, p. 49-56, 2016.

SCHLIEMANN, A. D.; CARRAHER, D. W.; BRIZUELA, B. M.; EARNEST, D.; GOODROW, A.; LARA-ROTH, S. Algebra in elementary school. En: PATEMAN, N.; DOUGHERTY, G.; ZILLIOX, J. (Eds.). Proceedings of the 27th Conference of the International Group for the Psychology of Mathematics Education and the 25th Conference of Psychology of Mathematics Education North America. Honolulu: CRDG, College of Education, University of Hawaii, 2003. p. 127-134.

SHIELD, M.; DOLE, S. Assessing the potential of mathematics textbooks to promote deep learning. Educational Studies in Mathematics, Dordrecht, v. 82, n. 2, p. 183-199, 2013. 
STYLIANIDES, G. J. Reasoning-and-proving in school mathematics textbooks. Mathematical thinking and learning, Philadelphia, v. 11, n. 4, p. 258-288, 2009.

TARR, J. E.; CHÁVEZ, Ó.; REYS, R. E.; REYS, B. J. From the written to the enacted curricula: The intermediary role of middle school mathematics teachers in shaping students' opportunity to learn. School Science and Mathematics, Chicago, v. 106, n. 4, p. 191-201, 2006.

Submetido em 02 de Novembro de 2020. Aprovado em 07 de Junho de 2021. 